\title{
Rees products and lexicographic shellability
}

\author{
Svante Linusson* $^{*}$, John Shareshian ${ }^{\dagger}$ and Michelle L. Wachs ${ }^{\ddagger}$ \\ Dedicated to Adriano Garsia
}

\begin{abstract}
We use the theory of lexicographic shellability to provide various examples in which the rank of the homology of a Rees product of two partially ordered sets enumerates some set of combinatorial objects, perhaps according to some natural statistic on the set. Many of these examples generalize a result of J. Jonsson, which says that the rank of the unique nontrivial homology group of the Rees product of a truncated Boolean algebra of degree $n$ and a chain of length $n-1$ is the number of derangements in $\mathfrak{S}_{n}$.
\end{abstract}

1 Introduction

Part I. Lexicographical shellability $\quad 249$

2 Edge labelings of Rees products $\quad 249$

3 Ascent-free chains of $P * T_{t, n}$

Part II. Applications

4 Symmetric function preliminaries

5 Chain product analog of $B_{n}$

$6 q$-analog of $B_{n}$

$7 p$-analog of chain product analog of $B_{n}$

8 The noncrossing partition lattice

${ }^{*}$ Royal Swedish Academy of Sciences Research Fellow supported by a grant from the Knut and Alice Wallenberg Foundation.

${ }^{\dagger}$ Supported in part by NSF Grants DMS 0604233 and 0902142.

${ }^{\ddagger}$ Supported in part by NSF Grants DMS 0604562 and 0902323. 


\section{Introduction}

Rees products of posets were defined and studied by A. Björner and V. Welker in [7]. While the main results in [7] provide combinatorial analogues of constructions in commutative algebra, it has turned out that Rees products of certain posets are connected with permutation enumeration and permutation statistics. The first indication of this connection is provided by a conjecture in [7], which says that the reduced Euler characteristic of the order complex of the Rees product of the truncated Boolean algebra $B_{n} \backslash\{\emptyset\}$ and a chain of length $n-1$ is the number of derangements in the symmetric group $\mathfrak{S}_{n}$. This conjecture was proved by J. Jonsson in [12].

As we shall describe below, generalizations of Jonsson's result, along with similar results, have been proved. Our purposes in this paper are

1. to give additional examples of Rees products whose order complexes have reduced Euler characteristics that enumerate certain classes of combinatorial objects, possibly according to some natural statistic, and

2. to show how the theory of lexicographic shellability applies to certain Rees products, in particular relating the homology of the order complex of the Rees product of a lexicographically shellable poset $P$ with a poset whose Hasse diagram is a rooted $t$-ary tree to the homology of the order complexes of some rank-selected subposets of $P$.

These two purposes are in fact intertwined. We prove all of our results on reduced Euler characteristics of order complexes of Rees products using lexicographic shellings.

All posets studied in this paper are finite. We call a poset $P$ semipure if for each $x \in P$, the lower order ideal $P_{\leq x}:=\{y \in P: y \leq x\}$ is pure, that is, any two maximal chains in $P_{\leq x}$ have the same length. The $\operatorname{rank}_{P}(x)$ of such an element $x$ is the length of a maximal chain in $P_{\leq x}$. Given semipure posets $P, Q$ with respective rank functions $r_{P}, r_{Q}$, the Rees product $P * Q$ is the poset whose underlying set is

$$
\left\{(p, q) \in P \times Q: r_{P}(p) \geq r_{Q}(q)\right\},
$$

with order relation given by $\left(p_{1}, q_{1}\right) \leq\left(p_{2}, q_{2}\right)$ if and only if all of the conditions

- $p_{1} \leq_{P} p_{2}$,

- $q_{1} \leq_{Q} q_{2}$, and

- $r_{P}\left(p_{1}\right)-r_{P}\left(p_{2}\right) \geq r_{Q}\left(q_{1}\right)-r_{Q}\left(q_{2}\right)$ 


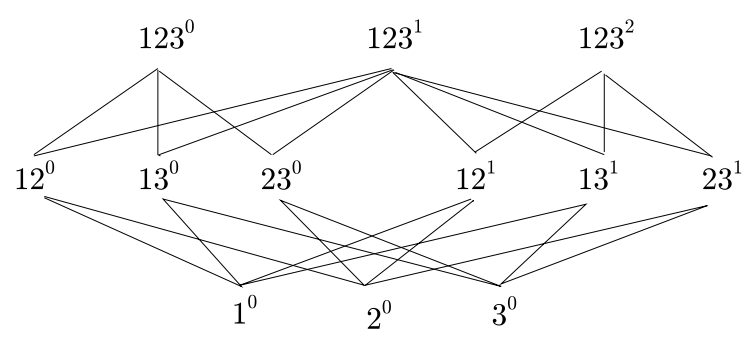

Figure 1: $\left(B_{3} \backslash\{\emptyset\}\right) * C_{2}$.

hold. In other words, $\left(p_{2}, q_{2}\right)$ covers $\left(p_{1}, q_{1}\right)$ in $P * Q$ if and only if

1. $p_{2}$ covers $p_{1}$ in $P$, and

2. either $q_{2}=q_{1}$ or $q_{2}$ covers $q_{1}$ in $Q$.

In Figure 1, the Rees product of the truncated Boolean algebra $B_{3} \backslash\{\emptyset\}$ and the chain $C_{2}:=\{0<1<2\}$ is given. The element $(S, j)$ is written as $S^{j}$ with the set brackets and commas omitted.

For any poset $P$, the order complex $\Delta P$ is the abstract simplicial complex whose $k$-dimensional faces are chains (totally ordered subsets) of length $k$ from $P$. A simplicial complex $\Delta$ is Cohen-Macaulay if for each face $F \in \Delta$ (including the empty face), the reduced (integral, simplicial) homology of the link $l k_{\Delta}(F)$ is trivial in all dimensions except possibly $\operatorname{dim}\left(l k_{\Delta}(F)\right.$ ). Every Cohen-Macaulay complex is pure, that is, all maximal faces of a CohenMacaulay complex have the same dimension. A poset is said to be CohenMacaulay if its order complex is Cohen-Macaulay. We will say that a poset has a particular topological property if its order complex has that property. The (reduced) homology of $P$ is given by $\tilde{H}_{k}(P):=\tilde{H}_{k}(\Delta P ; \mathbb{Z})$. For further information on Cohen-Macaulay posets, see the surveys given in [3, 24, 28].

Björner and Welker [7, Corollary 2] prove that the Rees product of any Cohen-Macaulay poset with any acyclic Cohen-Macaualy poset is CohenMacaulay. It is known that both $B_{n}^{-}:=B_{n} \backslash\{\emptyset\}$ and the chain $C_{n}$ of length $n$ are Cohen-Macaulay, and $C_{n}$ is acyclic. Thus the result of Jonsson mentioned above says that, with $d_{n}$ denoting the number of derangements in $\mathfrak{S}_{n}$,

$$
\mathrm{rk} \widetilde{H}_{n-1}\left(B_{n}^{-} * C_{n-1}\right)=d_{n} .
$$

Generalizations of (1.1) appear in the paper [21] of Shareshian and Wachs. For a poset $P$ with unique minimum element $\hat{0}, P^{-}$will denote 
$P \backslash\{\hat{0}\}$. For a prime power $q>1$ and a positive integer $n$, the poset of all subspaces of an $n$-dimensional vector space over the $q$-element field $\mathbb{F}_{q}$ will be denoted by $B_{n}(q)$. Also, $\mathcal{D}_{n}$ will denote the set of all derangements in $\mathfrak{S}_{n}$. It is shown in [21] that

$$
\operatorname{rk} \tilde{H}_{n-1}\left(B_{n}(q)^{-} * C_{n-1}\right)=\sum_{\sigma \in \mathcal{D}_{n}} q^{\left(\begin{array}{c}
n \\
2
\end{array}\right)-\operatorname{maj}(\sigma)+\operatorname{exc}(\sigma)},
$$

where maj and exc are, respectively, the major index and the excedance number, introduced by MacMahon in [15, Vol. I, pp. 135, 186; Vol. 2, p. viii], [16] in the early part of the 20th century and extensively studied thereafter.

A generalization of (1.2) appears in [21]. For positive integers $t, n$, let $T_{t, n}$ be the poset whose Hasse diagram is a complete $t$-ary tree of height $n$ with root at the bottom. To put it more formally, $T_{t, n}$ consists of all sequences of elements of $[t]:=\{1, \ldots, t\}$ that have length at most $n$, including the empty sequence. Given two such sequences $\mathrm{a}=\left(a_{1}, \ldots, a_{k}\right)$ and $\mathrm{b}=\left(b_{1}, \ldots, b_{l}\right)$, we declare that $\mathrm{a} \leq \mathrm{b}$ if $k \leq l$ and $a_{i}=b_{i}$ for all $i \in[k]$. Note that $T_{1, n}=C_{n}$.

It is shown in [21] that if $P$ is Cohen-Macaulay of length $n$ then so is $P * T_{t, n}$. Equation (1.4) below is proved in [21], and equation (1.3) follows quickly from (1.4) and [21, Corollary 2.4]. We have

$$
\operatorname{rk} \tilde{H}_{n-1}\left(B_{n}(q)^{-} * T_{t, n-1}\right)=t \sum_{\sigma \in \mathcal{D}_{n}} q^{\left(\begin{array}{c}
n \\
2
\end{array}\right)-\operatorname{maj}(\sigma)+\operatorname{exc}(\sigma)} t^{\operatorname{exc}(\sigma)}
$$

and

$$
\mathrm{rk} \tilde{H}_{n-1}\left(\left(B_{n}(q) * T_{t, n}\right)^{-}\right)=t \sum_{\sigma \in \mathfrak{S}_{n}} q^{\left(\begin{array}{l}
n \\
2
\end{array}\right)-\operatorname{maj}(\sigma)+\operatorname{exc}(\sigma)} t^{\operatorname{exc}(\sigma)}
$$

One can also find in [21] type BC analogues of the results mentioned above, where $B_{n}$ and $B_{n}(q)$ are replaced, respectively, by the poset of faces of the $n$-crosspolytope and the poset of totally isotropic subspaces of a $2 n$ dimensional vector space over $\mathbb{F}_{q}$ equipped with a nondegenerate alternating bilinear form, and $\mathcal{D}_{n}$ is replaced by the set of elements of the Weyl group of type BC that act as derangements on the set of vertices of the crosspolytope. In [17], P. Muldoon and M. Readdy prove an analog of (1.1) that involves the poset of faces of the $n$-cube.

As was mentioned above, the results of Björner and Welker [7] are concerned with Cohen-Macaulayness of Rees products. It turns out that analogous results for lexicographic shellability can be obtained and utilized to 
obtain enumerative results. Definitions and basic facts about lexicographic shellability are given in Section 2.

Let $P$ be a pure poset of length $n$. For $S \subseteq[0, n]:=\{0,1, \ldots, n\}$, the rank-selected subposet $P_{S}$ is the subposet of $P$ consisting of all $x \in X$ satisfying $r_{P}(x) \in S$. If $P$ is lexicographically shellable then $P$ is CohenMacaulay, as is every rank-selected subposet of $P$ (cf. [2]). Thus, for all $S \subseteq[0, n]$, the homology of $P_{S}$ is determined by the Betti number

$$
\beta\left(P_{S}\right):=\mathrm{rk} \tilde{H}_{|S|-1}\left(P_{S}\right) .
$$

Let $\hat{0}_{T}$ be the minimum element of $T_{t, n}$. Note that if $P$ has a unique minimum element $\hat{0}_{P}$ then the poset $P * T_{t, n}$ has a minimum element $\left(\hat{0}_{P}, \hat{0}_{T}\right)$, but no maximum element. Write $\left(P * T_{t, n}\right)^{+}$for the poset $P * T_{t, n}$ with a maximum element appended. In Section 2 we show that if $P$ is lexicographically shellable then so is $\left(P * T_{t, n}\right)^{+}$for all $t$. (In fact, we prove a stronger result; see Theorem 2.3.)

We call $S \subseteq \mathbb{N}$ stable if there is no $i \in \mathbb{N}$ such that $\{i, i+1\} \subseteq S$. For $X \subseteq \mathbb{N}$, we write $\mathcal{P}_{\text {stab }}(X)$ for the set of all stable $S \subseteq X$. We use the lexicographic shellings described in Section 2 to prove in Section 3 that, for pure, lexicographically shellable $P$ of length $n$,

$$
\beta\left(\left(P * T_{t, n}\right)^{-}\right)=\sum_{S \in \mathcal{P}_{\text {stab }}([n-2])} \beta\left(P_{[n-1] \backslash S}\right) t^{|S|+1}(1+t)^{n-2|S|-1} .
$$

In fact, we prove in Section 3 several formulae similar to (1.5) involving either $P^{-} * T_{t, n}$ or $\left(P * T_{t, n}\right)^{-}$. (More general versions of these formulae in which the only requirement on $\mathrm{P}$ is that it be pure will appear in a forthcoming paper.)

In Sections 5, 6, 7 and 8 we apply our results from Sections 2 and 3 to obtain enumerative results.

The Boolean algebra $B_{n}$ is the direct product of $n$ copies of the chain $C_{1}$. In Section 5 we prove generalizations of the $q=1$ cases of (1.3) and (1.4) in which we replace $B_{n}$ with an arbitrary product of finite chains. Let $\mu=\left(\mu_{1}, \ldots, \mu_{k}\right)$ be a weak composition of $n$ into $k$ parts, that is a $k$-tuple of nonnegative integers whose sum is $n$. The product poset $B_{\mu}:=\prod_{i=1}^{k} C_{\mu_{i}}$ is pure of length $n$. It is well known that $B_{\mu}$ is lexicographically shellable.

Let $M(\mu)$ be the multiset in which each $i \in[k]$ appears with multiplicity $\mu_{i}$. A multiset permutation of $M(\mu)$ is a $2 \times n$ array $\left(a_{i j}\right)$ such that

- the multisets $\left\{a_{1 j}: j \in[n]\right\}$ and $\left\{a_{2 j}: j \in[n]\right\}$ are both equal to $M(\mu)$,

- $a_{1, j} \leq a_{1, j+1}$ for all $j \in[n-1]$. 
Let $w=\left(a_{i j}\right)$ be a multiset permutation of $M(\mu)$. We say $w$ is multiset derangement of $M(\mu)$ if

- $a_{1 j} \neq a_{2 j}$, for all $j \in[n]$.

We say $w$ is a Smirnov word on $M(\mu)$ if

- $a_{2 j} \neq a_{2, j+1}$ for all $j \in[n-1]$.

An excedance of $w$ is any $j \in[n-1]$ such that $a_{2 j}>a_{1 j}$. A descent of $w$ is any $j \in[n-1]$ such that $a_{2 j}>a_{2, j+1}$. We write $\operatorname{EXC}(w)$ for the set of excedances of $w, \operatorname{DES}(w)$ for the set of descents of $w$, and $\operatorname{des}(w)$ and $\operatorname{exc}(w)$, respectively, for $|\operatorname{DES}(w)|$ and $|\operatorname{EXC}(w)|$.

Our main results in Section 5 say that if $\mathcal{M D}_{M(\mu)}$ and $\mathcal{S} \mathcal{W}_{M(\mu)}$ are, respectively, the sets of multiset derangements and Smirnov words on $M(\mu)$ then, for all $t \in \mathbb{P}$,

$$
\beta\left(B_{\mu}^{-} * T_{t, n-1}\right)=\sum_{w \in \mathcal{M} \mathcal{D}_{M(\mu)}} t^{1+\operatorname{exc}(w)},
$$

and

$$
\beta\left(\left(B_{\mu} * T_{t, n}\right)^{-}\right)=\sum_{w \in \mathcal{S} \mathcal{W}_{M(\mu)}} t^{1+\operatorname{des}(w)} .
$$

When $M(\mu)$ is the set $[n]$, equation (1.6) is the $q=1$ case of (1.3). Since des and exc are equidistributed on the symmetric group $\mathfrak{S}_{n}$, equation (1.7) is the $q=1$ case of (1.4).

In Section 6 we revisit the Rees products $B_{n}(q) * T_{t, n}$ that were studied in [21]. Comparing (1.4) with a formula for $\mathrm{rk} \tilde{H}_{n-1}\left(\left(B_{n}(q) * T_{t, n}\right)^{-}\right)$obtained using the techniques developed herein, we exhibit a permutation statistic called aid such that the pair (aid, des) is equidistributed on $\mathfrak{S}_{n}$ with the pair (maj, exc).

In Section 7 we aim for $p$-analogues of the results in Section 5 . Given a weak composition $\mu=\left(\mu_{1}, \ldots, \mu_{k}\right)$ of $n$, a natural choice for a $p$-analogue to the poset $B_{\mu}$ is the lattice $B_{\mu}(p)$ of subgroups of the abelian $p$-group $\bigoplus_{j=1}^{k} \mathbb{Z} / p^{\mu_{j}} \mathbb{Z}$. We examine $B_{\mu}(p)^{-} * T_{t, n-1}$ and $\left(B_{\mu}(p) * T_{t, n}\right)^{-}$. Here our results are less than optimal. We show that there exist statistics $s_{1}, s_{2}$ : $\mathbb{P}^{n} \rightarrow \mathbb{N}$ such that

$$
\beta\left(B_{\mu}(p)^{-} * T_{t, n-1}\right)=\sum_{w \in \mathcal{M} \mathcal{D}_{M(\mu)}} p^{s_{1}(w)} t^{1+\operatorname{exc}(w)}
$$


and

$$
\beta\left(\left(B_{\mu}(p) * T_{t, n}\right)^{-}\right)=\sum_{w \in \mathcal{S} \mathcal{W}_{M(\mu)}} p^{s_{2}(w)} t^{1+\operatorname{des}(w)}
$$

However, we lack natural combinatorial interpretations for $s_{1}$ and $s_{2}$.

In Section 8 we consider the lattice $\mathrm{NC}_{n}$ of noncrossing partitions of $[n]$, which is known to be lexicographically shellable. We show that

$$
\beta\left(\left(\mathrm{NC}_{n+1} * T_{t, n}\right)^{-}\right)=\frac{1}{n+1} \sum_{k=0}^{n-1}\left(\begin{array}{c}
n-1 \\
k
\end{array}\right) \sum_{w \in[n+1]^{n-k}} t^{\operatorname{des}(w)+k}
$$

and

$$
\begin{aligned}
& \beta\left(\mathrm{NC}_{n+1}^{-} * T_{t, n-1}\right) \\
& \quad=(-1)^{n}+\frac{1}{n+1} \sum_{r=0}^{n-1}(-1)^{r}\left(\begin{array}{c}
n+1 \\
r
\end{array}\right) \sum_{k=0}^{n-1-r}\left(\begin{array}{c}
n-1-r \\
k
\end{array}\right) \sum_{w \in[n+1]^{n-k-r}} t^{\operatorname{des}(w)+k} .
\end{aligned}
$$

Equation (1.10) reduces to a particularly nice enumerative formula when $t$ is set equal to 1 , namely

$$
\beta\left(\left(\mathrm{NC}_{n+1} * C_{n}\right)^{-}\right)=(n+2)^{n-1} .
$$

Proofs of the various identities stated above involve symmetric function formulae for generating functions for words with no double descent, words with no double ascent, Smirnov words, and multiset derangements, keeping track of descents, ascents, descents and excedances, respectively. The formula involving Smirnov words follows from work in [21], while the remaining formulae are due to Ira Gessel. We give all of these formulae in Section 4.

\section{Part I.}

\section{Lexicographical shellability}

\section{Edge labelings of Rees products}

After reviewing some basic facts from the theory of lexicographic shellability (cf. [2, 4-6, 28]), we will present our main results on lexicographic shellability 
of Rees products. Let $P$ be a bounded poset, i.e., a poset with a unique minimum element and a unique maximum element, and let $\operatorname{Cov}(P)$ be the set of pairs $(x, y) \in P \times P$ such that $y$ covers $x$ in $P$. Let $L$ be another poset and let $W$ be the set of all finite sequences of elements of $L$. The given partial ordering of $L$ induces a lexicographic ordering $\preceq$ on $W$, which is also a partial order. An edge labeling of $P$ by $L$ is a function $\lambda: \operatorname{Cov}(P) \rightarrow L$. Given such a function $\lambda$ and a saturated chain $C=\left\{x_{1}<\cdots<x_{m}\right\}$ from $P$, we write $\lambda(C)$ for $\left(\lambda\left(x_{1}, x_{2}\right), \ldots, \lambda\left(x_{m-1}, x_{m}\right)\right) \in W$. An ascent in $C$ is any $i \in[m-1]$ satisfying $\lambda\left(x_{i}, x_{i+1}\right) \leq \lambda\left(x_{i+1}, x_{i+2}\right)$. We say $\lambda$ is weakly increasing on $C$ if each $i \in[m-1]$ is an ascent in $C$. The edge labeling $\lambda$ is an EL-labeling of $P$ if whenever $x<y$ in $P$ there is a unique maximal chain $C$ in the interval $[x, y]$ on which $\lambda$ is weakly increasing and for all other maximal chains $D$ in $[x, y]$ we have $\lambda(C) \prec \lambda(D)$. A bounded poset that admits an EL-labeling is said to be EL-shellable.

The notion of EL-shellability for pure posets was introduced by Björner in [2]. A more general concept called CL-shellability, introduced by Björner and Wachs in [4], also associates label sequences with maximal chains of a poset. We will not define CL-labelings here. Both notions were subsequently extended to all bounded posets by Björner and Wachs in [6]. All of our results in this section and the next section hold for CL-labelings as well as EL-labelings. For the sake of simplicity we state and prove them only for EL-labelings. The proofs for CL-labelings are virtually the same as those for EL-labelings.

Given an EL-labeling $\lambda$ on $P$, we call a maximal chain $C$ from $P$ ascentfree if its label sequence contains no ascent. The descent set of a maximal chain $x_{0}<x_{1}<\cdots<x_{n}$ is defined to be the set $\left\{i \in[n-1]: \lambda\left(x_{i-1}, x_{i}\right) \not \mathbf{Z}\right.$ $\left.\lambda\left(x_{i}, x_{i+1}\right)\right\}$. Thus a maximal chain is ascent-free if and only if its descent set is $[n-1]$.

One of the main results in the theory of lexicographic shellability is the following result.

Theorem 2.1. (Björner and Wachs [6]) Let $\lambda$ be an EL-labeling of a bounded poset $P$ with minimum $\hat{0}$ and maximum $\hat{1}$. Then $P \backslash\{\hat{0}, \hat{1}\}$ is homotopy equivalent to a wedge of spheres, where for each $k \in \mathbb{N}$ the number of spheres of dimension $(k-2)$ is the number of ascent-free maximal chains of length $k$.

We will also need the following basic result. Given a pure poset $P$ of length $n$ and a set $S \subseteq[0, n]$, recall that the rank selected subposet is defined by

$$
P_{S}:=\left\{x \in P: r_{P}(x) \in S\right\} .
$$


Theorem 2.2. (Björner [2]) Let $\lambda$ be an EL-labeling of a bounded pure poset $P$ of length $n$. For $S \subseteq[n-1]$, let $c(S)$ be the number of maximal chains in $P$ having descent set $S$ with respect to $\lambda$. Then $P_{S}$ has the homotopy type of a wedge of $c(S)$ spheres of dimension $|S|-1$.

Given a poset $P$, by $\hat{P}$ we mean the poset $P$ with a new minimum element $\hat{0}$ and a new maximum element $\hat{1}$ attached even if $P$ already has such elements. Given a poset $P$ with a minimum element $\hat{0}$, we say that an edge labeling $\lambda: \operatorname{Cov}(P) \rightarrow L$ is a semi-EL-labeling if $[\hat{0}, m]$ is an ELlabeling for each maximal element $m$ of $P$. Note if $P$ is bounded then $\lambda$ is a semi-EL-labeling if and only if it is an EL-labeling. Recall that we defined $T_{t, n}$ to be the poset whose Hasse diagram is the complete $t$-ary tree of height $n$ with the root at the bottom. The edge labeling in which all the edges in $\operatorname{Cov}\left(T_{t, n}\right)$ are labeled with 1 is clearly a semi-EL-labeling of $T_{t, n}$.

Theorem 2.3. Let $P_{1}$ and $P_{2}$ be semipure posets of the same length. Assume also that $P_{2}$ has a minimum element $\hat{0}_{2}$. Let $\lambda_{1}: \operatorname{Cov}\left(\hat{P}_{1}\right) \rightarrow L_{1}$ be an ELlabeling of $\hat{P}_{1}$ and let $\lambda_{2}: \operatorname{Cov}\left(P_{2}\right) \rightarrow L_{2}$ be a semi-EL-labeling of $P_{2}$. Let $\hat{0}_{1}$ denote the minimum element of $\hat{P}_{1}$ and let $\hat{1}_{1}$ denote the maximum element. Let $\left(\hat{0}_{1}, \hat{0}_{2}\right)$ denote the minimum element of $\widehat{P_{1} * P_{2}}$ and let $\hat{1}$ denote the maximum element. Define the edge labeling

$$
\lambda: \operatorname{Cov}\left({\widehat{P}+P_{2}}_{2}\right) \rightarrow L_{1} \times\left(L_{2} \uplus\left\{\hat{0}_{L_{2}}\right\}\right)
$$

by

$$
\lambda((x, k),(y, l))= \begin{cases}\left(\lambda_{1}(x, y), \lambda_{2}(k, l)\right) & \text { if } k<_{P_{2}} l \\ \left(\lambda_{1}(x, y), \hat{0}_{L_{2}}\right) & \text { if } k=l\end{cases}
$$

for $(y, l)<\hat{1}$, and

$$
\lambda((x, k), \hat{1})=\left(\lambda_{1}\left(x, \hat{1}_{1}\right), \hat{0}_{L_{2}}\right) .
$$

Then $\lambda$ is an EL-labeling of $\widehat{P_{1} * P_{2}}$.

Proof. Case 1: $(x, k)<(y, l)<\hat{1}$ in $\widehat{P_{1} * P_{2}}$. Then $x<y$ in $\hat{P}_{1}$ and $k \leq l$ in $P_{2}$. It follows that there is a unique maximal chain $\left\{x=u_{0}<\cdots<u_{m}=\right.$ $y\}$ in $[x, y]$ on which $\lambda_{1}$ is weakly increasing and a unique maximal chain $\left\{k=c_{0}<\cdots<c_{r(l)-r(k)}=l\right\}$ in $[k, l]$ on which $\lambda_{2}$ is weakly increasing. Let

$$
e_{i}= \begin{cases}k & \text { for } 0 \leq i \leq m+r(k)-r(l) \\ c_{i-m-r(k)+r(l)} & \text { for } m+r(k)-r(l)<i \leq m .\end{cases}
$$


The labeling $\lambda$ is weakly increasing on the maximal chain

$$
C:=\left\{\left(u_{0}, e_{0}\right)<\left(u_{1}, e_{1}\right)<\cdots<\left(u_{m}, e_{m}\right)\right\}
$$

of the interval $I:=[(x, k),(y, l)]$.

To establish uniqueness of the maximal chain with weakly increasing labels, suppose that $\lambda$ is weakly increasing on the maximal chain $D=$ $\left\{\left(v_{0}, f_{0}\right)<\cdots<\left(v_{m}, f_{m}\right)\right\}$ in the interval $I$. Then $\lambda_{1}$ is weakly increasing on the chain $\left\{v_{0}<\cdots<v_{m}\right\}$, which implies that $v_{i}=u_{i}$ for all $0 \leq i \leq m$. Moreover, if $\lambda(D)=\left(\left(a_{1}, d_{1}\right), \ldots,\left(a_{m}, d_{m}\right)\right)$ then we must have $d_{i}=\hat{0}_{L_{2}}$ for $1 \leq i \leq m+r(k)-r(l)$ and $d_{i} \leq d_{i+1}$ in $L_{2}$ for $m+r(k)-r(l)<i \leq m-1$. If $d_{i}=\hat{0}_{L_{2}}$ then $f_{i-1}=f_{i}$, and if $d_{i} \in L_{2}$ then $f_{i-1}$ is covered by $f_{i}$ in $P_{2}$ and $d_{i}=\lambda_{2}\left(f_{i-1}, f_{i}\right)$. It follows that if $j=m+r(k)-r(l)$ then

$$
k=f_{0}=f_{1}=\cdots=f_{j}
$$

and

$$
\left\{f_{j}<f_{j+1}<\cdots<f_{m}\right\}
$$

is the unique maximal chain of the interval $[k, l]$ in $P_{2}$ for which $\lambda_{2}$ is weakly increasing. Thererfore $f_{i}=e_{i}$ for all $i$.

Next we show that the maximal chain $C$ of $I$ has a label sequence that lexicographically precedes the label sequences of all maximal chains of $I$. Let $D=\left\{\left(v_{0}, f_{0}\right)<\cdots<\left(v_{m}, f_{m}\right)\right\}$ be another maximal chain in $I$. Assume that $\left(u_{i}, e_{i}\right)=\left(v_{i}, f_{i}\right)$ for $0 \leq i<t$ but $\left(u_{t}, e_{t}\right) \neq\left(v_{t}, f_{t}\right)$. We need to show that

$$
\lambda\left(\left(u_{t-1}, e_{t-1}\right),\left(u_{t}, e_{t}\right)\right)<\lambda\left(\left(v_{t-1}, f_{t-1}\right),\left(v_{t}, f_{t}\right)\right)
$$

in $L_{1} \times\left(L_{2} \uplus\left\{\hat{0}_{L_{2}}\right\}\right)$

First we handle the case in which $1 \leq t \leq m+r(k)-r(l)$. In this case we have $e_{t-1}=e_{t}=k$, which implies

$$
\lambda\left(\left(u_{t-1}, e_{t-1}\right),\left(u_{t}, e_{t}\right)\right)=\left(\lambda_{1}\left(u_{t-1}, u_{t}\right), \hat{0}_{L_{2}}\right) .
$$

If $u_{t}=v_{t}$ then $f_{t} \neq e_{t}$, which implies that $f_{t}$ covers $f_{t-1}=k$ in $P_{2}$. Since $\lambda_{2}\left(f_{t-1}, f_{t}\right)>\hat{0}_{L_{2}}$ and

$$
\lambda\left(\left(v_{t-1}, f_{t-1}\right),\left(v_{t}, f_{t}\right)\right)=\left(\lambda_{1}\left(v_{t-1}, v_{t}\right), \lambda_{2}\left(f_{t-1}, f_{t}\right)\right),
$$

(2.1) holds. Now assume $u_{t} \neq v_{t}$. We have

$$
\lambda_{1}\left(u_{t-1}, u_{t}\right)<\lambda_{1}\left(v_{t-1}, v_{t}\right)
$$


in $L_{1}$. Indeed, it is a basic property of EL-labelings that if $P$ is a poset with EL-labeling $\lambda$ then for each interval $[x, y]$, if $a$ covers $x$ in the unique maximal chain of $[x, y]$ with weakly increasing labels and $b$ is an atom of $[x, y]$ other than $a$, then $\lambda(x, a)<\lambda(x, b)$ (cf. [2, Proposition 2.5], [6, Lemma 5.3]). Since $\lambda\left(\left(v_{t-1}, f_{t-1}\right),\left(v_{t}, f_{t}\right)\right)=\left(\lambda_{1}\left(v_{t-1}, v_{t}\right), d\right)$, for some $d \in L_{2} \uplus \hat{0}_{L_{2}}$, the desired inequality (2.1) follows from (2.2) and (2.3).

Now assume $m+r(k)-r(l)<t \leq m$. In this case $u_{t}$ and $v_{t}$ cover $u_{t-1}=v_{t-1}$ in $\hat{P}_{1}$, and $e_{t}$ and $f_{t}$ cover $e_{t-1}=f_{t-1}$ in $P_{2}$. It now follows from the basic property of EL-labelings mentioned in the previous paragraph that either (2.3) and $\lambda_{2}\left(e_{t-1}, e_{t}\right) \leq \lambda_{2}\left(f_{t-1}, f_{t}\right)$ or $\lambda_{1}\left(u_{t-1}, u_{t}\right) \leq \lambda_{1}\left(v_{t-1}, v_{t}\right)$ and $\lambda_{2}\left(e_{t-1}, e_{t}\right)<\lambda_{2}\left(f_{t-1}, f_{t}\right)$ hold, which yields the desired conclusion (2.1).

Case 2: $(x, k)<\hat{1}$ in $\widehat{P_{1} * P_{2}}$. Then $x \leq \hat{1}_{1}$ in $\hat{P}_{1}$ and there is a unique maximal chain $\left\{x=u_{0}<\cdots<u_{m}<\hat{1}_{1}\right\}$ in $\left[x, \hat{1}_{1}\right]$ on which $\lambda_{1}$ is weakly increasing. The labeling $\lambda$ is weakly increasing on the maximal chain

$$
C:=\left\{\left(u_{0}, k\right)<\cdots<\left(u_{m}, k\right)<\hat{1}\right\}
$$

of the interval $[(x, k), \hat{1}]$. To establish uniqueness of the maximal chain with weakly increasing labels, note that the top label of every maximal chain of $[(x, k), \hat{1}]$ is of the form $\left(\lambda_{1}\left(v, \hat{1}_{1}\right), \hat{0}_{L_{2}}\right)$, where $v$ is a maximal element of $P_{1}$. Hence if $D=\left\{\left(v_{0}, f_{0}\right)<\cdots<\left(v_{m^{\prime}}, f_{m^{\prime}}\right)<\hat{1}\right\}$ is a maximal chain of $[(x, k), \hat{1}]$ with weakly increasing labels then

$$
\lambda(D)=\left\{\left(a_{1}, \hat{0}_{L_{2}}\right), \ldots,\left(a_{m^{\prime}+1}, \hat{0}_{L_{2}}\right)\right\},
$$

where $a_{1} \leq \cdots \leq a_{m^{\prime}+1}$ in $L_{1}$. It follows that $f_{i}=k$ for all $i=1, \ldots, m^{\prime}$ and $\left\{v_{0}<\cdots<v_{m^{\prime}}<\hat{1}_{1}\right\}$ is the unique maximal chain of $\left[x, \hat{1}_{1}\right]$ with weakly increasing labels. Hence $m=m^{\prime}$ and $v_{i}=u_{i}$ for all $i=1, \ldots, m$.

Now let $D=\left\{\left(v_{0}, f_{0}\right)<\cdots<\left(v_{m^{\prime}}, f_{m^{\prime}}\right)<\hat{1}\right\}$ be a maximal chain in $[(x, k), \hat{1}]$ that is different from $C$. We show that the label sequence of $C$ is lexicographically less than that of $D$. Assume that $\left(u_{i}, k\right)=\left(v_{i}, f_{i}\right)$ for $1 \leq i<t$ but $\left(u_{t}, k\right) \neq\left(v_{t}, f_{t}\right)$. We need to show that

$$
\lambda\left(\left(u_{t-1}, k\right),\left(u_{t}, k\right)\right)<\lambda\left(\left(v_{t-1}, f_{t-1}\right),\left(v_{t}, f_{t}\right)\right)
$$

in $L_{1} \times\left(L_{2} \uplus\left\{\hat{0}_{L_{2}}\right\}\right)$. If $u_{t}=v_{t}$ then $f_{t}$ covers $f_{t-1}=k$ in $P_{2}$. We have

$$
\begin{aligned}
\lambda\left(\left(u_{t-1}, k\right),\left(u_{t}, k\right)\right) & =\left(\lambda_{1}\left(u_{t-1}, u_{t}\right), \hat{0}_{L_{2}}\right) \\
& =\left(\lambda_{1}\left(v_{t-1}, v_{t}\right), \hat{0}_{L_{2}}\right) \\
& <\left(\lambda_{1}\left(v_{t-1}, v_{t}\right), \lambda_{2}\left(f_{t-1}, f_{t}\right)\right) \\
& =\lambda\left(\left(v_{t-1}, f_{t-1}\right),\left(v_{t}, f_{t}\right)\right) .
\end{aligned}
$$


If $u_{t} \neq v_{t}$ then by the basic property of EL-labelings mentioned above, $\lambda_{1}\left(u_{t-1}, u_{t}\right)<\lambda_{1}\left(v_{t-1}, v_{t}\right)$. It follows that

$$
\begin{aligned}
\lambda\left(\left(u_{t-1}, k\right),\left(u_{t}, k\right)\right) & =\left(\lambda_{1}\left(u_{t-1}, u_{t}\right), \hat{0}_{L_{2}}\right) \\
& <\left(\lambda_{1}\left(v_{t-1}, v_{t}\right), \lambda_{2}\left(f_{t-1}, f_{t}\right)\right) \\
& =\lambda\left(\left(v_{t-1}, f_{t-1}\right),\left(v_{t}, f_{t}\right)\right) .
\end{aligned}
$$

\section{Ascent-free chains of $P * T_{t, n}$}

Let $P$ be a semipure poset of length $n$. Let $\lambda_{P}: \operatorname{Cov}(\hat{P}) \rightarrow L_{P}$ be an EL-labeling of $\hat{P}$ and let $\lambda_{T}$ be the semi-EL-labeling of $T_{t, n}$ in which each edge has label 1 . In this section we count the ascent-free maximal chains of $\widehat{P * T_{t, n}}$ under the EL-labeling $\lambda: \operatorname{Cov}\left(\widehat{P * T_{t, n}}\right) \rightarrow\left(L_{P} \times\{0<1\}\right)$ described in Theorem 2.3.

For $j=0, \ldots, m$, let $S_{m, j}$ be the set of sequences $\left(d_{1}, \ldots, d_{m}\right) \in\{0,1\}^{m}$ such that $\sum_{i=1}^{m} d_{i}=j$. Given any maximal chain $D=\left\{\left(\hat{0}_{P}, \hat{0}_{T}\right)<\left(x_{0}, f_{0}\right)<\right.$ $\left.\cdots<\left(x_{m}, f_{m}\right)<\hat{1}\right\}$ of $\widehat{P * T_{t, n}}$, we have that $\left\{x_{0}<x_{1}<\cdots<x_{m}\right\}$ is a maximal chain of $P$ and $\left(r\left(f_{1}\right)-r\left(f_{0}\right), r\left(f_{2}\right)-r\left(f_{1}\right), \ldots, r\left(f_{m}\right)-r\left(f_{m-1}\right)\right) \in$ $S_{m, j}$, for some $j$. Conversely, given any maximal chain $C=\left\{x_{0}<x_{1}<\right.$ $\left.\cdots<x_{m}\right\}$ of $P$ and any $d \in S_{m, j}$, there is a maximal chain $D=\left\{\left(\hat{0}_{P}, \hat{0}_{T}\right)<\right.$ $\left.\left(x_{0}, f_{0}\right)<\cdots<\left(x_{m}, f_{m}\right)<\hat{1}\right\}$ of $\widehat{P * T_{t, n}}$ such that $r\left(f_{i}\right)-r\left(f_{i-1}\right)=d_{i}$ for all $i \in[m]$. Let $[C, d]$ be the set of all such maximal chains of $\widehat{P * T_{t, n}}$.

The following propositions clearly hold.

Proposition 3.1. The sets $[C, d]$, where $C$ is a maximal chain of $P$ of length $m$ and $d \in S_{m, j}$ for $j=0, \ldots, m$, partition the set of maximal chains of $\widehat{P * T_{t, n}}$. Moreover if $d \in S_{m, j}$ then $|[C, d]|=t^{j}$.

Proposition 3.2. Let

$$
C:=\left\{x_{0}<\cdots<x_{m}\right\}
$$

be a maximal chain of $P$ and let $d:=\left(d_{1}, \ldots, d_{m}\right) \in\{0<1\}^{m}$. Then for each maximal chain $D \in[(C, d)]$ we have

$$
\begin{aligned}
\lambda(D)= & \left(\left(\lambda_{P}\left(\hat{0}_{P}, x_{0}\right), 0\right),\left(\lambda_{P}\left(x_{0}, x_{1}\right), d_{1}\right), \ldots,\right. \\
& \left.\left(\lambda_{P}\left(x_{m-1}, x_{m}\right), d_{m}\right),\left(\lambda_{P}\left(x_{m}, \hat{1}_{P}\right), 0\right)\right) .
\end{aligned}
$$

Consequently, $D$ is ascent-free if and only if $\lambda_{P}\left(\hat{0}_{P}, x_{0}\right) \not \lambda_{P}\left(x_{0}, x_{1}\right)$ and

$$
\forall i \in[m], \quad \lambda_{P}\left(x_{i-1}, x_{i}\right) \leq \lambda_{P}\left(x_{i}, x_{i+1}\right) \Longrightarrow d_{i}=1 \quad \text { and } \quad d_{i+1}=0
$$

holds. Here we have set $x_{m+1}:=\hat{1}_{P}$ and $d_{m+1}:=0$. 
Given a word $w=w_{1} \cdots w_{n}$ over a partially ordered alphabet $A$, we say $i \in[n-1]$ is an ascent of $w$ if $w_{i} \leq w_{i+1}$ and that $i \in[n-2]$ is a double ascent if $w_{i} \leq w_{i+1} \leq w_{i+2}$. Let $\operatorname{asc}(w)$ denote the number of ascents of $w$ and

$$
\operatorname{NDA}_{n}(A):=\left\{w \in A^{n}: w \text { has no double ascents }\right\} .
$$

We are now ready to count the ascent-free maximal chains. We begin with the case in which the semipure poset $P$ has a unique maximum element. In this case $P$ must necessarily be a pure poset of length $n$. All maximal chains of $\hat{P}$ have length $n+2$ and must have an ascent at $n+1$ under the EL-labeling. We leave it to the reader to observe that Propositions 3.1 and 3.2 imply the following result.

Theorem 3.3. If $P$ has a unique maximum element then the number of ascent-free maximal chains of $\widehat{P * T_{t, n}}$ of length $n+2$ under the EL-labeling of Theorem 2.3 is given by

$$
\sum_{\substack{w \in \mathrm{NDA}_{n+1}\left(L_{P}\right) \\ w_{1} \nless w_{2} \\ w_{n} \succeq w_{n+1}}} c(w) t^{\operatorname{asc}(w)+1}(1+t)^{n-1-2 \operatorname{asc}(w)},
$$

where $c(w)$ is the number of maximal chains of $P \uplus \hat{0}_{P}$ with label sequence $w$.

In the general case in which it is not assumed that $P$ has a unique maximum element, we have the following result, which also is a consequence of Propositions 3.1 and 3.2.

Theorem 3.4. Let $m \in \mathbb{N}$. Then the number ascent-free maximal chains of $\widehat{P * T_{t, n}}$ of length $m+2$ under the EL-labeling of Theorem 2.3 is given by

$$
\begin{aligned}
& \sum_{\substack{w \in \mathrm{NDA}_{m+2}\left(L_{P}\right) \\
w_{1} \nless w_{2} \\
w_{m+1} \leq w_{m+2}}} c(w) t^{\operatorname{asc}(w)}(1+t)^{m-2 \operatorname{asc}(w)} \\
& +\sum_{\substack{w \in \mathrm{NDA}_{m+2}\left(L_{P}\right) \\
w_{1} \nless w_{2} \\
w_{m+1} \leq w_{m+2}}} c(w) t^{\operatorname{asc}(w)}(1+t)^{m+1-2 \operatorname{asc}(w)},
\end{aligned}
$$

where $c(w)$ is the number of maximal chains of $\hat{P}$ of length $m+2$ with label sequence $w$. 
Note that if $P$ has a unique minimum element then $P * T_{t, n}$ has unique minimum element, which implies that $P * T_{t, n}$ is contractible. Hence the number of ascent-free maximal chains of $\widehat{P * T_{t, n}}$ has to be 0 . This is corroborated by $c(w)=0$ if $w_{1} \not \leq w_{2}$, which follows from the fact that there is only one maximal chain in each interval $[\hat{0}, a]$ of $\hat{P}$, where $a$ is an atom of $P$. Therefore in the case that $P$ has a unique minimum element, it is more interesting to consider the number of ascent-free chains of the interval $\left(P * T_{t, n}\right)^{+}$of $\widetilde{P * T_{t, n}}$. The following results also follow from Propositions 3.1 and 3.2 .

Theorem 3.5. If $P$ has both a unique minimum element and a unique maximum element then the number of ascent-free maximal chains of $(P *$ $\left.T_{t, n}\right)^{+}$under the EL-labeling of Theorem 2.3 is given by

$$
\sum_{\substack{w \in \operatorname{NDA}_{n}\left(L_{P}\right) \\ w_{n-1} \not w_{n}}} c(w) t^{\operatorname{asc}(w)+1}(1+t)^{n-1-2 \operatorname{asc}(w)},
$$

where $c(w)$ is the number of maximal chains of $P$ with label sequence $w$.

Theorem 3.6. Let $m \in \mathbb{N}$. If $P$ has a unique minimum element then the number of ascent-free maximal chains of $\left(P * T_{t, n}\right)^{+}$of length $m+1$ under the EL-labeling of Theorem 2.3 is given by

$$
\begin{aligned}
& \sum_{\substack{w \in \mathrm{NDA}_{m+1}\left(L_{P}\right) \\
w_{m} \leq w_{m+1}}} c(w) t^{\operatorname{asc}(w)}(1+t)^{m-2 \operatorname{asc}(w)} \\
& +\sum_{\substack{w \in \mathrm{NDA}_{m+1}\left(L_{P}\right) \\
w_{m} \leq w_{m+1}}} c(w) t^{\operatorname{asc}(w)}(1+t)^{m+1-2 \operatorname{asc}(w)},
\end{aligned}
$$

where $c(w)$ is the number of maximal chains of $P^{+}$of length $m+1$ with label sequence $w$.

For pure $P$ we can restate the above results by applying Theorem 2.2. We need to recall the following terminology and notation. A set of integers is stable if it contains no two consecutive integers. For $X \subseteq \mathbb{Z}$, the set of all stable subsets of $X$ is denoted by $\mathcal{P}_{\text {stab }}(X)$. For $i \leq j \in \mathbb{N}$, let $[i, j]:=$ $\{i, i+1, \ldots, j\}$ and $[j]:=[1, j]$. If $P$ is a poset of length $n$ let

$$
\beta(P):=\operatorname{rk} \tilde{H}_{n}(P)
$$


If $P$ has a unique minimum element $0 \hat{~ l e t ~}$

$$
P^{-}:=P \backslash\{\hat{0}\} .
$$

Corollary 3.7. Let $P$ be a pure poset of length $n$ such that $\hat{P}$ is EL-shellable. Assume that $P$ has a unique maximum element. Then

$$
\beta\left(P * T_{t, n}\right)=\sum_{S \in \mathcal{P}_{\text {stab }}([n-2])} \beta\left(P_{[0, n-1] \backslash S}\right) t^{|S|+1}(t+1)^{n-2|S|-1} .
$$

If $P$ also has a unique minimum element then

$$
\beta\left(\left(P * T_{t, n}\right)^{-}\right)=\sum_{S \in \mathcal{P}_{\text {stab }}([n-2])} \beta\left(P_{[n-1] \backslash S}\right) t^{|S|+1}(t+1)^{n-2|S|-1} .
$$

Corollary 3.8. Let $P$ be a pure poset of length $n$ such that $\hat{P}$ is EL-shellable. Then

$$
\begin{aligned}
\beta(P & \left.* T_{t, n}\right) \\
= & \sum_{S \in \mathcal{P}_{\text {stab }}([n-1])} \beta\left(P_{[0, n] \backslash S)} t^{|S|}(t+1)^{n-2|S|}\right. \\
& +\sum_{S \in \mathcal{P}_{\text {stab }}([n-2])} \beta\left(P_{[0, n-1] \backslash S}\right) t^{|S|+1}(t+1)^{n-2|S|-1} .
\end{aligned}
$$

If $P$ has a unique minimum element then

$$
\begin{aligned}
\beta( & \left.\left(P * T_{t, n}\right)^{-}\right) \\
= & \sum_{S \in \mathcal{P}_{\text {stab }}([n-1])} \beta\left(P_{[n] \backslash S}\right) t^{|S|}(t+1)^{n-2|S|} \\
& +\sum_{S \in \mathcal{P}_{\text {stab }}([n-2])} \beta\left(P_{[n-1] \backslash S}\right) t^{|S|+1}(t+1)^{n-2|S|-1} .
\end{aligned}
$$

\section{Part II.}

\section{Applications}

\section{Symmetric function preliminaries}

Let $h_{n}=h_{n}\left(x_{1}, x_{2}, \ldots\right)$ denote the complete homogenous symmetric function of degree $n$ in indeterminants $\mathbf{x}:=x_{1}, x_{2}, \ldots$ and $e_{n}=e_{n}\left(x_{1}, x_{2}, \ldots\right)$ 
denote the elementary symmetric function of degree $n$ in indeterminants $\mathbf{x}$. That is

$$
h_{n}(\mathbf{x}):=\sum_{1 \leq i_{i} \leq \cdots \leq i_{n}} x_{i_{1}} \cdots x_{i_{n}} \quad \text { and } \quad e_{n}(\mathbf{x}):=\sum_{1 \leq i_{i}<\cdots<i_{n}} x_{i_{1}} \cdots x_{i_{n}} .
$$

Also let

$$
[n]_{t}:=1+t+\cdots+t^{n-1}
$$

In this section we will discuss various combinatorial interpretations of variations of the symmetric function

$$
\frac{\sum_{i \geq 0} h_{i} z^{i}}{1-\sum_{i \geq 2} t[i-1]_{t} h_{i} z^{i}}
$$

which play a key role in the proofs of the results in the subsequent sections. These and other interpretations are discussed in [20, Section 7].

Let $w=w_{1} \cdots w_{n} \in \mathbb{P}^{n}$. Recall that we say $i \in[n-1]$ is an ascent of $w$ if $w_{i} \leq w_{i+1}$ and that $i \in[n-2]$ is a double ascent if $w_{i} \leq w_{i+1} \leq w_{i+2}$. Recall that $\operatorname{asc}(w)$ denotes the number of ascents of $w$ and

$$
\mathrm{NDA}_{n}:=\operatorname{NDA}_{n}(\mathbb{P})=\left\{w \in \mathbb{P}^{n}: w \text { has no double ascents }\right\}
$$

Similarly, $i \in[n-1]$ is a descent of $w$ if $w_{i}>w_{i+1}$ and $i \in[n-2]$ is a double descent if $w_{i}>w_{i+1}>w_{i+2}$. Let $\operatorname{des}(w)$ denote the number of descents of $w$ and

$$
\mathrm{NDD}_{n}:=\operatorname{NDD}_{n}(\mathbb{P})=\left\{w \in \mathbb{P}^{n}: w \text { has no double descents }\right\}
$$

We write $\mathbf{x}_{w}$ for $x_{w_{1}} \cdots x_{w_{n}}$.

We begin by presenting the following interpretations due to Gessel, see Theorem 7.3 of [20]. (Gessel's original proofs will appear in [11].)

$$
\begin{aligned}
& 1+\sum_{n \geq 1} z^{n} \sum_{\substack{w \in \mathrm{NDA}_{n} \\
w_{1}>w_{2} \\
w_{n-1}>w_{n}}} t^{\operatorname{asc}(w)}(1+t)^{n-2-2 \operatorname{asc}(w)} \mathbf{x}_{w}=\frac{1}{1-\sum_{i \geq 2} t[i-1]_{t} e_{i} z^{i}} \\
& 1+\sum_{n \geq 1} z^{n} \sum_{\substack{w \in \mathrm{NDA}_{n} \\
w_{n-1}>w_{n}}} t^{\operatorname{asc}(w)}(1+t)^{n-1-2 \operatorname{asc}(w)} \mathbf{x}_{w}=\frac{\sum_{i \geq 0} e_{i} z^{i}}{1-\sum_{i \geq 2} t[i-1]_{t} e_{i} z^{i}}
\end{aligned}
$$




$$
1+\sum_{n \geq 1} z^{n} \sum_{\substack{w \in \mathrm{NDD}_{n} \\ w_{1} \leq w_{2} \\ w_{n}-1 \leq w_{n}}} t^{\operatorname{des}(w)}(1+t)^{n-2-2 \operatorname{des}(w)} \mathbf{x}_{w}=\frac{1}{1-\sum_{i \geq 2} t[i-1]_{t} h_{i} z^{i}}
$$

$$
1+\sum_{n \geq 1} z^{n} \sum_{\substack{w \in \mathrm{NDD}_{n} \\ w_{n-1} \leq w_{n}}} t^{\operatorname{des}(w)}(1+t)^{n-1-2 \operatorname{des}(w)} \mathbf{x}_{w}=\frac{\sum_{i \geq 0} h_{i} z^{i}}{1-\sum_{i \geq 2} t[i-1]_{t} h_{i} z^{i}}
$$

Next we present an interpretation due to Shareshian and Wachs [20]. A barred word of length $n$ over alphabet $A$ is an element of $(A \times\{0,1\})^{n}$. We visualize barred words as words over $A$ in which some of the letters are barred; $(a, 1)$ is a barred letter and $(a, 0)$ is an unbarred letter. If $w$ is a barred word then $|w|$ denotes the word $w$ with the bars removed. Similarly, let $|a|=|\bar{a}|=a$. If $\alpha$ is a barred or unbarred letter, we refer to $|\alpha|$ as the absolute value of $\alpha$. For a barred word $w$, let $\operatorname{bar}(w)$ denote the number of barred letters of $w$. Let $W_{n}$ be the set of barred words $w=w_{1} \cdots w_{n}$ of length $n$ over $\mathbb{P}$ satisfying

1. $w_{n}$ is unbarred

2. for all $i \in[n-1]$, if $\left|w_{i}\right|<\left|w_{i+1}\right|$ then $w_{i}$ is unbarred

3. for all $i \in[n-1]$, if $\left|w_{i}\right|>\left|w_{i+1}\right|$ then $w_{i}$ is barred.

Elements of $W_{n}$ are called banners in [20, Section 3], where it is shown that

$$
1+\sum_{n \geq 1} z^{n} \sum_{w \in W_{n}} t^{\operatorname{bar}(w)} \mathbf{x}_{|w|}=\frac{\sum_{i \geq 0} h_{i} z^{i}}{1-\sum_{i \geq 2} t[i-1]_{t} h_{i} z^{i}} .
$$

We will also need an interpretation due to Askey and Ismail [1] and one due to Stanley (personal communication, see Theorem 7.2 of [20]). Given a finite multiset $M$ over $\mathbb{P}$, let $\mathfrak{S}_{M}$ denote the set of multiset permutations of $M$. Recall that we can write $w \in \mathfrak{S}_{M}$ in two-line notation as a $2 \times|M|$ array $\left(w_{i, j}\right)$ whose top row is a weakly increasing arrangement of the multiset $M$ and whose bottom row is an arbitrary arrangement of $M$. By supressing the top row, we write $w$ in one-line notation as the word, $w_{1} \cdots w_{|M|}$, where $w_{i}:=w_{2, i}$. If $w \in \mathfrak{S}_{M}$ we say that $w$ has length $|M|$. An excedance of a multiset permutation $w=\left(w_{i, j}\right)$, written in two-line notation, is a column $j$ such that $w_{1, j}<w_{2, j}$. Let exc $(w)$ be the number of excedances of $w$.

Recall that $w=\left(w_{i, j}\right) \in \mathfrak{S}_{M}$ is a multiset derangement if each of the columns of $w$ have distinct entries, i.e., $w_{1, j} \neq w_{2, j}$ for all $j=1, \ldots,|M|$. For example, if 


$$
w=\left[\begin{array}{lllllll}
1 & 1 & 1 & 2 & 3 & 3 & 4 \\
3 & 2 & 3 & 1 & 4 & 1 & 1
\end{array}\right]
$$

then $w$ is a multiset derangement in $\mathfrak{S}_{\left\{1^{3}, 2,3^{2}, 4\right\}}$ and $\operatorname{exc}(w)=4$.

Now let $\mathcal{M D}_{n}$ be the set of all multiset derangements of length $n$. Askey and Ismail [1] (see also [13]) proved the following t-analog of MacMahon's [15, Sec. III, Ch. III] result on multiset derangements

$$
\sum_{n \geq 0} z^{n} \sum_{w \in \mathcal{M} \mathcal{D}_{n}} t^{\operatorname{exc}(w)} \mathbf{x}_{w}=\frac{1}{1-\sum_{i \geq 2} t[i-1]_{t} e_{i} z^{i}}
$$

Recall from Section 1 that a multiset permutation $w=w_{1} \cdots w_{n} \in \mathfrak{S}_{M}$ is called a Smirnov word if it has no adjacent repeats, i.e. $w_{i} \neq w_{i+1}$ for all $i=1, \ldots, n-1$. Let $\mathcal{S} \mathcal{W}_{n}$ be the set of all Smirnov words of length $n$. Stanley (see Theorem 7.2 and (7.7) of [20]) observed that the following t-analog of a result of Carlitz, Scoville and Vaughan [9]

$$
\sum_{n \geq 0} z^{n} \sum_{w \in \mathcal{S} \mathcal{W}_{n}} t^{\operatorname{des}(w)} \mathbf{x}_{w}=\frac{\sum_{i \geq 0} e_{i} z^{i}}{1-\sum_{i \geq 2} t[i-1]_{t} e_{i} z^{i}}
$$

is equivalent to (4.5) by $\mathrm{P}$-partition reciprocity [25, Section 4.5].

\section{Chain product analog of $B_{n}$}

In this section we generalize the $q=1$ case of (1.3) and (1.4) by utilizing the results of the previous section. Given a weak composition $\mu:=\left(\mu_{1}, \ldots, \mu_{k}\right)$ of $n$, let $B_{\mu}$ denote the product of chains $C_{\mu_{1}} \times \cdots \times C_{\mu_{k}}$. Recall that $M(\mu)$ denotes the multiset $\left\{1^{\mu_{1}}, \ldots, k^{\mu_{k}}\right\}$. Given a multiset $M$, let $\mathcal{M D}_{M}$ be the set of multiset derangements of the multiset $M$ and let $\mathcal{S} \mathcal{W}_{M}$ be the set of Smirnov words that are multiset permutations of $M$.

Theorem 5.1. Let $\mu$ be a composition of $n$. Then $B_{\mu}^{-} * T_{t, n-1}$ and $\left(B_{\mu} * T_{t, n}\right)^{-}$ have the homotopy type of a wedge of $(n-1)$-spheres. The numbers of spheres in these wedges are, respectively,

$$
\beta\left(B_{\mu}^{-} * T_{t, n-1}\right)=\sum_{w \in \mathcal{M} \mathcal{D}_{M(\mu)}} t^{\operatorname{exc}(w)+1}
$$

and

$$
\beta\left(\left(B_{\mu} * T_{t, n}\right)^{-}\right)=\sum_{w \in \mathcal{S} \mathcal{W}_{M(\mu)}} t^{\operatorname{des}(w)+1}
$$


Proof. We begin by applying Theorem 3.3 to $P:=B_{\mu}^{-}$, which has length $n-1$. Let $k=l(\mu)$. There is a well known EL-labeling of $B_{\mu}$ in which the edge

$$
\left(\left(x_{1}, \ldots, x_{i}, \ldots, x_{k}\right),\left(x_{1}, \ldots, x_{i}+1, \ldots, x_{k}\right)\right)
$$

is labeled by $i$. Here $L_{P}$ is the totally ordered set $\{1<2<\cdots<k\}$. Hence $B_{\mu}^{-} * T_{t, n-1}$ has an EL-labeling as described in Theorem 2.3. The label sequence of each maximal chain of $P \uplus\{\hat{0}\}=B_{\mu}$ is a permutation of the multiset $M(\mu)$. Moreover each mulitset permutation occurs exactly once as the label sequence of a maximal chain. So $c(w)=1$ if $w \in \mathfrak{S}_{M(\mu)}$ and $c(w)=0$ if $w \in[k]^{n}-\mathfrak{S}_{M(\mu)}$. It follows from Theorem 3.3 that the number of ascent-free maximal chains of $\widehat{B_{\mu}^{-} \widehat{* T_{t, n-1}}}$ under the given labeling is

$$
\sum_{\substack{w \in \mathrm{NDA}_{n} \cap \mathfrak{S}_{M(\mu)} \\ w_{1}>w_{2} \\ w_{n-1}>w_{n}}} t^{\operatorname{asc}(w)+1}(1+t)^{n-2-2 \operatorname{asc}(w)} .
$$

Similarly by Theorems 2.3 and 3.5 with $P=B_{\mu}$, the poset $\left(B_{\mu} * T_{t, n}\right)^{+}$has an EL-labeling for which the number of ascent-free maximal chains is

$$
\sum_{\substack{w \in \mathrm{NDA}_{n} \cap \mathfrak{S}_{M(\mu)} \\ w_{n-1}>w_{n}}} t^{\operatorname{asc}(w)+1}(1+t)^{n-1-2 \operatorname{asc}(w)} .
$$

Hence by Theorem 2.1, the posets $B_{\mu}^{-} * T_{t, n-1}$ and $\left(B_{\mu} * T_{t, n}\right)^{-}$have the homotopy type of a wedge of $(n-1)$-spheres and the top Betti numbers are given by

$$
\beta\left(B_{\mu}^{-} * T_{t, n-1}\right)=\sum_{\begin{array}{c}
w \in \mathrm{NDA}_{n} \cap \mathfrak{S}_{M(\mu)} \\
w_{1}>w_{2} \\
w_{n-1}>w_{n}
\end{array}} t^{\operatorname{asc}(w)+1}(1+t)^{n-2-2 \operatorname{asc}(w)},
$$

and

$$
\beta\left(\left(B_{\mu} * T_{t, n}\right)^{-}\right)=\sum_{\substack{w \in \mathrm{NDA}_{n} \cap \mathfrak{S}_{M(\mu)} \\ w_{n-1}>w_{n}}} t^{\operatorname{asc}(w)+1}(1+t)^{n-1-2 \operatorname{asc}(w)} .
$$

By combining (4.6) and (4.1) we obtain

$$
\sum_{w \in \mathcal{M} \mathcal{D}_{M}} t^{\operatorname{exc}(w)}=\sum_{\substack{w \in \mathrm{NDA}_{n} \cap \mathfrak{S}_{M} \\ w_{1}>w_{2} \\ w_{n-1}>w_{n}}} t^{\operatorname{asc}(w)}(1+t)^{n-2-2 \operatorname{asc}(w)},
$$


and by combining (4.7) and (4.2) we obtain

$$
\sum_{w \in \mathcal{S} \mathcal{W}_{M}} t^{\operatorname{des}(w)}=\sum_{\substack{w \in \mathrm{NDA}_{n} \cap \mathfrak{S}_{M} \\ w_{n-1}>w_{n}}} t^{\operatorname{asc}(w)}(1+t)^{n-1-2 \operatorname{asc}(w)}
$$

for all multisets $M$ on $\mathbb{P}$ of size $n$. Equations (5.1) and (5.2) now follow from (5.3) and (5.4), respectively.

Remark 5.2. When $M=\left\{1^{n}\right\}$, equation (5.4) reduces to a result of Foata and Schützenberger [10], which is used to show that the Eulerian polynomials are palindromic and unimodal. We see from (5.3) and (5.4), respectively, that the polynomials $\sum_{w \in \mathcal{M} \mathcal{D}_{M}} t^{\operatorname{exc}(w)}$ and $\sum_{w \in \mathcal{S} \mathcal{W}_{M}} t^{\operatorname{des}(w)}$ are palindromic and unimodal for all multisets $M$.

\section{6. q-analog of $B_{n}$}

The lattice $B_{n}(q)$ of subspaces of an $n$-dimensional vector space over the finite field $\mathbb{F}_{q}$ is bounded and pure of length $n$. It is well known that $B_{n}(q)$ is EL-shellable (see [28]). Using (3.3) to compute $\beta\left(\left(B_{n}(q) * T_{t, n}\right)^{-}\right)$and equating the resulting formula with the formula given in (1.4), we obtain a new Mahonian permutation statistic, which we call aid, and we show that the pairs (aid, des) and (maj, exc) are equidistributed on $\mathfrak{S}_{n}$.

Let $\sigma \in \mathfrak{S}_{n}$. Recall that an inversion of $\sigma$ is a pair $(\sigma(i), \sigma(j))$ such that $1 \leq i<j \leq n$ and $\sigma(i)>\sigma(j)$. An admissible inversion of $\sigma$ is an inversion $(\sigma(i), \sigma(j))$ that satisfies either

- $1<i$ and $\sigma(i-1)<\sigma(i)$ or

- there is some $k$ such that $i<k<j$ and $\sigma(i)<\sigma(k)$.

We write $\operatorname{inv}(\sigma)$ for the number of inversions of $\sigma$ and ai $(\sigma)$ for the number of admissible inversions of $\sigma$. For example, if $\sigma=6431275$ then there are 11 inversions, but only $(6,5)$ and $(7,5)$ are admissible. So $\operatorname{inv}(\sigma)=11$ and $\operatorname{ai}(\sigma)=2$.

Now let

$$
\operatorname{aid}(\sigma):=\operatorname{ai}(\sigma)+\operatorname{des}(\sigma)
$$

It turns out that aid is equidistributed with the Mahonian permutation statistics inv and maj on $\mathfrak{S}_{n}$. We give a short combinatorial proof of this in Proposition 6.3 below. First we prove the following more general joint distribution result. 
Theorem 6.1. For all $n \geq 0$,

$$
\sum_{\sigma \in \mathfrak{S}_{n}} q^{\operatorname{aid}(\sigma)} t^{\operatorname{des}(\sigma)}=\sum_{\sigma \in \mathfrak{S}_{n}} q^{\operatorname{maj}(\sigma)} t^{\operatorname{exc}(\sigma)} .
$$

Proof. It is well known (see [27, Theorem 3.12.3]) that for all $S \subseteq[n-1]$,

$$
\beta\left(B_{n}(q)_{S}\right)=\sum_{\substack{\sigma \in \mathfrak{S}_{n} \\ \operatorname{DES}(\sigma)=S}} q^{\operatorname{inv}(\sigma)} .
$$

Hence by (3.3) we have

$$
\begin{aligned}
\beta\left(\left(B_{n}(q) * T_{t, n}\right)^{-}\right) & =\sum_{\substack{S \in \mathcal{P}_{\text {stab }}([1, n-2]) \\
\operatorname{DES}(\sigma)=[1, n-1] \backslash S}} \sum_{\substack{\sigma \in \mathfrak{G}_{n} \\
(6.1)}} q^{\operatorname{inv}(\sigma)} t^{|S|+1}(t+1)^{n-1-2|S|} \\
& =\sum_{\substack{\sigma \in \mathfrak{S}_{n} \cap N_{n} \text { NA }_{n} \\
\sigma_{n-1}>\sigma_{n}}} q^{\operatorname{inv}(\sigma)} t^{\operatorname{asc}(\sigma)+1}(1+t)^{n-1-2 \operatorname{asc}(\sigma)} .
\end{aligned}
$$

We will rewrite the expression (6.1) as the enumerator of barred permutations. Given a set $X$ of size $n$, a barred permutation of $X$ is a word $w_{1} w_{2} \cdots w_{n}$ with $n$ distinct letters in $X$, in which some of the letters are barred. Let $\left|w_{i}\right|$ denote the letter $w_{i}$ with the bar removed if there is one and let $|w|=\left|w_{1}\right| \cdots\left|w_{n}\right| \in \mathfrak{S}_{X}$, where $\mathfrak{S}_{X}$ is the set of ordinary permutations of $X$. Let $\operatorname{bar}(w)$ denote the number of bars of $w$. Let $\mathcal{W}_{X}$ be the set of barred permutations $w$ of $X$ satisfying

(A) $w_{n}$ is barred

(B) if $i \in[n-1]$ and $\left|w_{i}\right|<\left|w_{i+1}\right|$ then $w_{i}$ is barred and $w_{i+1}$ is not barred.

It is not hard to see that the expression (6.1) equals

$$
\sum_{w \in \mathcal{W}_{[n]}} q^{\operatorname{inv}(|w|)} t^{\operatorname{bar}(w)}
$$

which by Lemma 6.2 below equals

$$
\sum_{\sigma \in \mathfrak{S}_{n}} q^{\left(\begin{array}{c}
n \\
2
\end{array}\right)-\mathrm{ai}(\sigma)} t^{\operatorname{des}(\sigma)+1} .
$$

Hence

$$
\beta\left(\left(B_{n}(q) * T_{t, n}\right)^{-}\right)=\sum_{\sigma \in \mathfrak{S}_{n}} q^{\left(\begin{array}{c}
n \\
2
\end{array}\right)-\operatorname{ai}(\sigma)} t^{\operatorname{des}(\sigma)+1} .
$$

The result now follows from (1.4). 
Given barred permutations $\alpha \in \mathcal{W}_{A}$ and $\beta \in \mathcal{W}_{B}$, where $A$ and $B$ are disjoint sets, let $\alpha \cdot \beta$ denote the barred permutation in $\mathcal{W}_{A \uplus B}$ obtained by concatenating the words $\alpha$ and $\beta$. Also let $\theta$ denote the empty word. We define a map

$$
\varphi: \biguplus_{\substack{X \subseteq \mathbb{P} \\|X|<\infty}} \mathcal{W}_{X} \rightarrow \biguplus_{\substack{X \subseteq \mathbb{P} \\|X|<\infty}} \mathfrak{S}_{X}
$$

recursively as follows. If $w$ is in the domain of $\varphi$ and $m$ is the maximum letter of $|w|$ then

$$
\varphi(w)= \begin{cases}\theta & \text { if } w=\theta \\ m \cdot \varphi(\beta) & \text { if } w=\bar{m} \cdot \beta \\ \varphi(\beta) \cdot m \cdot \varphi(\alpha) & \text { if } w=\alpha \cdot m \cdot \beta \text { and } \beta \neq \theta\end{cases}
$$

Lemma 6.2. The map $\varphi$ is a well-defined bijection which satisfies

1. $\varphi\left(\mathcal{W}_{X}\right)=\mathfrak{S}_{X}$,

2. $\operatorname{des}(\varphi(w))+1=\operatorname{bar}(w)$,

3. $\operatorname{ai}(\varphi(w))=\left(\begin{array}{c}|X| \\ 2\end{array}\right)-\operatorname{inv}(|w|)$

for all finite nonempty subsets $X$ of $\mathbb{P}$ and all $w \in \mathcal{W}_{X}$.

Proof. By (B) of the definition of $\mathcal{W}_{X}$, if letter $m$ is barred in the word $w \in \mathcal{W}_{X}$ then it is the first letter of $w$. By $(\mathrm{A})$, if $m$ is unbarred it cannot be the last letter. Hence the three cases of the definition of $\varphi$ cover all possibilities. It is also clear from the definition of $\mathcal{W}_{X}$ that if $\alpha \cdot m \cdot \beta \in \mathcal{W}_{X}$ and $\beta \neq \theta$ then $\alpha \in \mathcal{W}_{A}$ and $\beta \in \mathcal{W}_{X \backslash(A \cup\{m\})}$ for some subset $A \subsetneq X$. Hence by induction on $|X|$ we have that $\varphi$ is a well-defined map that takes elements of $\mathcal{W}_{X}$ to $\mathfrak{S}_{X}$.

To show that $\varphi$ is a bijection satisfying (1) we construct its inverse. Define

$$
\psi: \biguplus_{\substack{X \subseteq \mathbb{P} \\|X|<\infty}} \mathfrak{S}_{X} \rightarrow \biguplus_{\substack{X \subseteq \mathbb{P} \\|X|<\infty}} \mathcal{W}_{X}
$$

recursively by

$$
\psi(\sigma)= \begin{cases}\theta & \text { if } \sigma=\theta \\ \bar{m} \cdot \psi(\delta) & \text { if } \sigma=m \cdot \delta \\ \psi(\delta) \cdot m \cdot \psi(\gamma) & \text { if } \sigma=\gamma \cdot m \cdot \delta \text { and } \gamma \neq \theta\end{cases}
$$


where $m$ is the maximum letter of $\sigma$. Let $\gamma \cdot m \cdot \delta \in \mathfrak{S}_{X}$. One can see that conditions (A) and (B) of the definition of $\mathcal{W}_{X}$ hold for $\psi(\gamma \cdot m \cdot \delta)$ whenever they hold for $\psi(\gamma)$ and $\psi(\delta)$. Hence by induction on $|X|, \psi$ is a well-defined map. One can easily also show by induction that $\varphi$ and $\psi$ are inverses of each other.

We also prove (2) by induction on $|X|$, with the base case $|X|=0$ being trivial. We do the third case of the definition of $\varphi$ and leave the second to the reader. Let $w=\alpha \cdot m \cdot \beta \in \mathcal{W}_{X}$ with $\beta \neq \theta$. If $\alpha \neq \theta$ then

$$
\operatorname{bar}(w)=\operatorname{bar}(\alpha)+\operatorname{bar}(\beta)=\operatorname{des}(\varphi(\alpha))+\operatorname{des}(\varphi(\beta))+2,
$$

by the induction hypothesis. Since $m$ is the largest element of $X$ and is not the last letter of $\varphi(w)$, we have

$$
\operatorname{des}(\varphi(w))=\operatorname{des}(\varphi(\beta))+1+\operatorname{des}(\varphi(\alpha)) .
$$

Hence (2) holds in this case.

Our proof of (3) proceeds by induction on $n=|X|$, the case $n=0$ being trivial.

If $w=\bar{m} \cdot \beta$ then

$$
\begin{aligned}
\operatorname{ai}(\varphi(w)) & =\operatorname{ai}(m \cdot \varphi(\beta)) \\
& =\operatorname{ai}(\varphi(\beta)) \\
& =\left(\begin{array}{c}
n-1 \\
2
\end{array}\right)-\operatorname{inv}(|\beta|) \\
& =\left(\begin{array}{c}
n \\
2
\end{array}\right)-(\operatorname{inv}(|\beta|)+n-1) \\
& =\left(\begin{array}{c}
n \\
2
\end{array}\right)-\operatorname{inv}(|\bar{m} \cdot \beta|) .
\end{aligned}
$$

Indeed, the first two equalities follow immediately from the definitions and the third follows from our inductive hypothesis.

Next, say $w=\alpha \cdot m \cdot \beta$ with $\alpha \in \mathcal{W}_{A}$ and $\beta \in \mathcal{W}_{B}$, where $|B|>0$. Set $\operatorname{inv}(A, B):=\mid\{(a, b): a \in A, b \in B, a>b\}$ It follows quickly from the inductive hypothesis and the definitions that 


$$
\begin{aligned}
\operatorname{ai}(\varphi(w))= & \operatorname{ai}(\varphi(\beta) \cdot m \cdot \varphi(\alpha)) \\
= & \operatorname{ai}(\varphi(\beta))+|A|+\operatorname{ai}(\varphi(\alpha))+\operatorname{inv}(B, A) \\
= & \left(\begin{array}{c}
|B| \\
2
\end{array}\right)-\operatorname{inv}(|\beta|)+n-1-|B| \\
& +\left(\begin{array}{c}
|A| \\
2
\end{array}\right)-\operatorname{inv}(|\alpha|)+|A||B|-\operatorname{inv}(A, B)
\end{aligned}
$$

Now

$$
\operatorname{inv}(|\alpha \cdot m \cdot \beta|)=\operatorname{inv}(|\alpha|)+|B|+\operatorname{inv}(|\beta|)+\operatorname{inv}(A, B)
$$

and a straightforward calculation shows that

$$
\left(\begin{array}{c}
|B| \\
2
\end{array}\right)+n-1+\left(\begin{array}{c}
|A| \\
2
\end{array}\right)+|A||B|=\left(\begin{array}{c}
n \\
2
\end{array}\right)
$$

Hence

$$
\operatorname{ai}(\varphi(w))=\left(\begin{array}{l}
n \\
2
\end{array}\right)-\operatorname{inv}(|\alpha \cdot m \cdot \beta|)
$$

as desired.

We pose the question of whether there is an enlightening direct bijective proof of Theorem 6.1. Our proof of Theorem 6.1 relies on (1.4), whose proof, in turn, relies on a $q$-analog of Euler's formula for the Eulerian polynomials derived by Shareshian and Wachs in [20]. A considerable amount of work in symmetric function theory and bijective combinatorics went into the proof of this $q$-analog of Euler's formula. Since the steps in deriving Theorem 6.1 from the $q$-analog of Euler's formula are reversible, a nice direct combinatorial proof of Theorem 6.1 would provide an interesting alternative proof of the $q$-analog of Euler's formula. Here we give a simple combinatorial proof that aid is Mahonian.

Proposition 6.3. Let $F_{n}(q)=\sum_{\sigma \in \mathfrak{S}_{n}} q^{\text {aid }(\sigma)}$. Then $F_{n}(q)$ satisfies the following recurrence for all $n \geq 2$,

$$
F_{n}(q):=(1+q) F_{n-1}(q)+\sum_{j=2}^{n-1}\left[\begin{array}{c}
n-1 \\
j-1
\end{array}\right]_{q} q^{j} F_{j-1}(q) F_{n-j}(q)
$$

Consequently $F_{n}(q)=[n]_{q} !$ 
Proof. The terms on the right side of the recurrence $q$-count permutations according to the position of $n$ in the permutation. That is for each $j$,

$$
\sum_{\substack{\sigma \in \mathfrak{S}_{n} \\
\sigma(n-j+1)=n}} q^{\operatorname{aid}(\sigma)}= \begin{cases}{\left[\begin{array}{c}
n-1 \\
j-1
\end{array}\right]_{q} q^{j} F_{j-1}(q) F_{n-j}(q)} & \text { if } j=2, \ldots, n-1 \\
F_{n-1}(q) & \text { if } j=1 \\
q F_{n-1}(q) & \text { if } j=n .\end{cases}
$$

It is easy to see that $[n]_{q}$ ! also satisfies the same recurrence relation.

A more natural Mahonian permutation statistic whose joint distribution with des is the same as that of aid is discussed in [22, 23]. This statistic is a member of a family of Mahonian statistics introduced by Rawlings [18].

\section{7. $p$-analog of chain product analog of $B_{n}$}

Given a prime $p$ and a weak composition $\mu:=\left(\mu_{1}, \ldots, \mu_{k}\right)$ of $n$, let $B_{\mu}(p)$ denote the lattice of subgroups of the abelian $p$-group $\mathbb{Z} / p^{\mu_{1}} \mathbb{Z} \times \cdots \times \mathbb{Z} / p^{\mu_{k}} \mathbb{Z}$. The poset $B_{\mu}(p)$ is a natural $p$-analog of $B_{\mu}$. It is pure and bounded of length $n$. Moreover, it provides the following $p$-analog of Theorem 5.1.

Theorem 7.1. Let $\mu$ be a weak composition of $n$ and let $p$ be a prime. Then $B_{\mu}(p)^{-} * T_{t, n-1}$ and $\left(B_{\mu}(p) * T_{t, n}\right)^{-}$have the homotopy type of a wedge of $(n-1)$-spheres. The numbers of spheres in these wedges are, respectively,

$$
\beta\left(B_{\mu}(p)^{-} * T_{t, n-1}\right)=\sum_{w \in \mathcal{M} \mathcal{D}_{M(\mu)}} p^{s_{1}(w)} t^{\operatorname{exc}(w)+1}
$$

and

$$
\beta\left(\left(B_{\mu}(p) * T_{t, n}\right)^{-}\right)=\sum_{w \in \mathcal{S} \mathcal{W}_{M(\mu)}} p^{s_{2}(w)} t^{\operatorname{des}(w)+1},
$$

where $s_{1}, s_{2}: \mathbb{P}^{n} \rightarrow \mathbb{N}$ are statistics on words over $\mathbb{P}$.

Proof. It is well known that $B_{\mu}(p)$ is EL-shellable. Hence by Theorem 2.3, $B_{n}(q)^{-} * T_{t, n-1}$ and $\left(B_{n}(q) * T_{t, n}\right)^{-}$are EL-shellable. It is also known (see $[8,(1.30)])$ that for all $S \subseteq[n-1]$,

$$
\beta\left(B_{\mu}(p)_{S}\right)=\sum_{\substack{w \in \mathfrak{S}_{M(\mu)} \\ \operatorname{DES}(w)=S}} p^{\operatorname{cocharge}(w)},
$$


where cocharge is a statistic on words introduced by Lascoux and Schützenberger for the purpose of showing that the Kostka polynomials have nonnegative integer coefficients. (We will not need the precise definition of cocharge here.)

Now by (3.2) we have

$$
\begin{aligned}
& \beta\left(B_{\mu}(p)^{-} * T_{t, n-1}\right) \\
& =\sum_{S \in \mathcal{P}_{\text {stab }}([2, n-2])} \sum_{\substack{w \in \mathfrak{S}_{M(\mu)} \\
\mathrm{DES}(w)=[1, n-1] \backslash S}} p^{\operatorname{cocharge}(w)} t^{|S|+1}(t+1)^{n-2-2|S|} .
\end{aligned}
$$

Hence

$$
\beta\left(B_{\mu}(p)^{-} * T_{t, n-1}\right)=\sum_{i=1}^{n} f_{i}(p) t^{i}
$$

where $f_{i}(p) \in \mathbb{N}[p]$. By $(5.3)$,

$$
f_{i}(1)=\left|\left\{w \in \mathcal{M D}_{M(\mu)}: \operatorname{exc}(w)=i-1\right\}\right|
$$

Since $f_{i}(1)$ is the sum of the coefficients of $f_{i}(p)$, we can assign a nonnegative integer $s_{1}(w)$ to each word $w$ in $\mathcal{M D}_{M(\mu)}$ so that

$$
f_{i}(p)=\sum_{\substack{w \in \mathcal{M} \mathcal{D}_{M(\mu)} \\ \operatorname{exc}(w)=i-1}} p^{s_{1}(w)}
$$

By plugging this into (7.3), we obtain the desired result (7.1).

The proof of (7.2) follows along the lines of that of (7.1) with (3.3) and (5.4) used instead of (3.2) and (5.3).

Problem. It would be interesting to find nice combinatorial descriptions of the coefficients of the polynomials $\beta\left(B_{\mu}(p)^{-} * T_{t, n-1}\right)$ and $\beta\left(\left(B_{\mu}(p) *\right.\right.$ $\left.\left.T_{t, n}\right)^{-}\right)$. That is, find natural statistics $s_{1}$ and $s_{2}$ for which (7.1) and (7.2) hold. When $w \in \mathfrak{S}_{M\left(1^{n}\right)}$, we see from (1.3) that $s_{1}(w)$ can be defined to be $\left(\begin{array}{l}n \\ 2\end{array}\right)-\operatorname{maj}(w)+$ exc and from $(6.2)$ that $s_{2}(w)$ can be defined to be $\left(\begin{array}{l}n \\ 2\end{array}\right)$ - ai.

\section{The noncrossing partition lattice}

A set partition $\pi$ is said to be noncrossing if for all $a<b<c<d$, whenever $a, c$ are in a block $B$ of $\pi$ and $b, d$ are in a block $B^{\prime}$ of $\pi$ then $B=B^{\prime}$. Let $\mathrm{NC}_{n}$ 
be the poset of noncrossing partitions of $[n]$ ordered by reverse refinement. This poset, known as the noncrossing partition lattice, was first introduced by Kreweras [14], who showed that it is a pure lattice with Möbius invariant equal to the signed Catalan number $(-1)^{n-1} \frac{1}{n}\left(\begin{array}{c}2 n-2 \\ n-1\end{array}\right)$. Björner and Edelman (cf. [2]) gave the first EL labeling of $\mathrm{NC}_{n}$ and later Stanley [26] gave a different EL-labeling in which the maximal chains are labeled with parking functions.

A word $w \in \mathbb{P}^{n}$ is said to be a parking function of length $n$ if its weakly increasing rearrangement $u$ satisfies $u_{i} \leq i$ for all $i \in[n]$. Let $\mathrm{PF}_{n}$ be the set of parking functions of length $n$. Recall that for $w=w_{1}, \ldots, w_{n} \in \mathbb{P}^{n}$,

$$
\operatorname{DES}(w):=\left\{i \in[n-1]: w_{i}>w_{i+1}\right\} \quad \text { and } \quad \operatorname{des}(w):=|\operatorname{DES}(w)| .
$$

Stanley uses his EL-labeling to prove that for all $S \subseteq[n-1]$,

$$
\beta\left(\left(\mathrm{NC}_{n+1}\right)_{[n-1] \backslash S}\right)=\left|\left\{w \in \mathrm{PF}_{n}: \operatorname{DES}(w)=S\right\}\right| .
$$

Theorem 8.1. For all $n, t \in \mathbb{P}$, the posets $\left(\mathrm{NC}_{n+1} * T_{t, n}\right)^{-}$and $\mathrm{NC}_{n+1}^{-} * T_{t, n-1}$ have the homotopy type of a wedge of $(n-1)$-spheres. The numbers of spheres in these wedges are, respectively,

$$
\beta\left(\left(\mathrm{NC}_{n+1} * T_{t, n}\right)^{-}\right)=\frac{1}{n+1} \sum_{k=0}^{n-1}\left(\begin{array}{c}
n-1 \\
k
\end{array}\right) \sum_{w \in[n+1]^{n-k}} t^{\operatorname{des}(w)+k}
$$

and

$$
\begin{aligned}
& \beta\left(\mathrm{NC}_{n+1}^{-} * T_{t, n-1}\right) \\
& \quad=(-1)^{n}+\frac{1}{n+1} \sum_{r=0}^{n-1}(-1)^{r}\left(\begin{array}{c}
n+1 \\
r
\end{array}\right) \sum_{k=0}^{n-1-r}\left(\begin{array}{c}
n-1-r \\
k
\end{array}\right) \sum_{w \in[n+1]^{n-k-r}} t^{\operatorname{des}(w)+k} .
\end{aligned}
$$

By a straightforward computation involving the binomial theorem, Theorem 8.1 reduces to the following result when $t=1$.

Corollary 8.2. For all $n \in \mathbb{P}$, the posets $\left(\mathrm{NC}_{n+1} * C_{n}\right)^{-}$and $\mathrm{NC}_{n+1}^{-} * C_{n-1}$ have the homotopy type of a wedge of $(n-1)$-spheres. The numbers of spheres in these wedges are, respectively,

$$
\beta\left(\left(\mathrm{NC}_{n+1} * C_{n}\right)^{-}\right)=(n+2)^{n-1}
$$


and

$$
\beta\left(\mathrm{NC}_{n+1}^{-} * C_{n-1}\right)=\frac{(n+1)^{n+1}+(-1)^{n}(n+3)}{(n+2)^{2}}
$$

Proof of Theorem 8.1. Since $\mathrm{NC}_{n+1}$ is EL-shellable it follows from Theorem 2.3 that $\mathrm{NC}_{n+1}^{-} * T_{t, n-1}$ and $\left(\mathrm{NC}_{n+1} * T_{t, n}\right)^{-}$have the homotopy type of a wedge of $(n-1)$-spheres.

Proof of (8.2). By substituting (8.1) into (3.3) we obtain

$$
\begin{aligned}
& \beta\left(\left(\mathrm{NC}_{n+1} * T_{t, n}\right)^{-}\right) \\
& \quad=\sum_{S \in \mathcal{P}_{\text {stab }}([1, n-2])}\left|\left\{w \in \mathrm{PF}_{n}: \operatorname{DES}(w)=S\right\}\right| t^{|S|+1}(t+1)^{n-2|S|-1} \\
& \quad=\sum_{\substack{w \in \mathrm{PF}_{n} \cap \mathrm{NDD}_{n} \\
w_{n-1} \leq w_{n}}} t^{\operatorname{des}(w)+1}(t+1)^{n-2 \operatorname{des}(w)-1}
\end{aligned}
$$

Let $\mathrm{WComp}_{n, k}$ be the set of all weak compositions of $n$ into $k$ parts. It is straightforward to show that $w \in \mathrm{PF}_{n}$ if and only if $w \in \mathfrak{S}_{M(\mu)}$ for some $\mu \in \mathrm{WComp}_{n, n}$ such that $\sum_{i=1}^{j} \mu_{i} \geq j$ for all $j=1, \ldots, n$. We will call a weak composition of $n$ into $n$ parts that satisfies this condition a parking composition of $n$, and let $\mathrm{PC}_{n}$ be the set of all parking compositions of $n$. It now follows from (8.4) that

$$
\beta\left(\left(\mathrm{NC}_{n+1} * T_{t, n}\right)^{-}\right)=\sum_{\mu \in \mathrm{PC}_{n}} \sum_{\substack{w \in \mathfrak{S}_{M(\mu)} \cap \mathrm{NDD}_{n} \\ w_{n-1} \leq w_{n}}} t^{\operatorname{des}(w)+1}(t+1)^{n-2 \operatorname{des}(w)-1}
$$

Note that every parking composition $\mu$ of $n$ can be viewed as an element of $\mathrm{WComp}_{n, n+1}$ by adjoining a 0 to the end of $\mu$. For $\mu, \mu^{\prime} \in \mathrm{WComp}_{n, n+1}$, we say that $\mu$ and $\mu^{\prime}$ are cyclically equivalent if $\mu^{\prime}$ can be obtained by cyclically rotating the parts of $\mu$. Since all elements of $\mathrm{WComp}_{n, n+1}$ are primitive words, i.e., they are not equal to a power of a shorter word, the equivalence classes of $\mathrm{WComp}_{n, n+1}$ under cyclic equivalence all have size equal to $n+1$. Moreover, each equivalence class has exactly one parking composition $\mu$, i.e. $\mu=\left(\mu_{1}, \ldots, \mu_{n}, 0\right)$ where $\left(\mu_{1}, \ldots, \mu_{n}\right) \in \mathrm{PC}_{n}$.

Given a weak composition $\mu$ of $n$, let

$$
F_{\mu}:=\sum_{\substack{w \in \mathfrak{S}_{M(\mu)} \cap \mathrm{NDD}_{n} \\ w_{n-1} \leq w_{n}}} t^{\operatorname{des}(w)+1}(t+1)^{n-2 \operatorname{des}(w)-1}
$$


and let

$$
\mathbf{x}^{\mu}:=x_{1}^{\mu_{1}} \cdots x_{k}^{\mu_{k}},
$$

for $\mu=\left(\mu_{1}, \ldots, \mu_{k}\right)$. It follows from (4.4) that $\sum_{\mu \in \mathrm{WComp}_{n, n+1}} F_{\mu} \mathbf{x}^{\mu}$, is a polynomial in $t$ whose coefficients are symmetric polynomials in the variables $x_{1}, \ldots, x_{n+1}$. Hence

$$
F_{\mu}=F_{\mu^{\prime}}
$$

whenever $\mu^{\prime}$ is a rearrangement of $\mu$; so $F_{\mu}$ is constant on cyclic equivalence classes of $\mathrm{WComp}_{n, n+1}$. We can therefore choose a representative of each cyclic equivalence class of $\mathrm{WComp}_{n, n+1}$ to compute the sum of $F_{\mu}$ over the weak compositions $\mu$ in $\mathrm{WComp}_{n, n+1}$. By letting the parking compositions be the chosen representatives, we arrive at

$$
\sum_{\mu \in \mathrm{WComp}_{n, n+1}} F_{\mu}=(n+1) \sum_{\mu \in \mathrm{PC}_{n}} F_{\mu}
$$

It now follows from (8.5) that

$$
\beta\left(\left(\mathrm{NC}_{n+1} * T_{t, n}\right)^{-}\right)=\frac{1}{n+1} \sum_{\mu \in \mathrm{WComp}_{n, n+1}} F_{\mu} .
$$

By combining (4.4) and (4.5) we have that for all $m, n \in \mathbb{P}$ and $\mu \in$ $\mathrm{WComp}_{n, m}$,

$$
F_{\mu}=\sum_{\substack{w \in W_{n} \\|w| \in \mathfrak{S}_{M(\mu)}}} t^{\operatorname{bar}(w)}
$$

which implies that

$$
\sum_{\mu \in \mathrm{WComp}_{n, m}} F_{\mu}=\sum_{\substack{w \in W_{n} \\|w| \in[m]^{n}}} t^{\operatorname{bar}(w)} .
$$

We claim that for all $m$ and $n$,

$$
\sum_{\substack{w \in W_{n} \\
|w| \in[m]^{n}}} t^{\operatorname{bar}(w)}=\sum_{k=0}^{n-1}\left(\begin{array}{c}
n-1 \\
k
\end{array}\right) t^{k} \sum_{u \in[m]^{n-k}} t^{\operatorname{des}(u)} .
$$

To prove this claim first note that there are two types of barred letters in $w \in W_{n}$. The type I barred letters are those that are followed by a letter 
equal to it in absolute value and the type II barred letters are those that are followed by a letter that is smaller than it in absolute value. The summand on the right side of the equation enumerates banners that have exactly $k$ barred letters of the first type. To obtain such a banner first choose the $k$ positions from the first $n-1$ positions in which the type I barred letters are to appear and leave them blank, then fill in the remaining $n-k$ positions with an arbitrary word $u$ in $[m]^{n-k}$, then fill in the $k$ barred positions that were left blank from right to left so that the letter equals its successor in absolute value, and finally put bars over the descent positions of the resulting word, the number of which is clearly equal to $\operatorname{des}(u)$.

By combining (8.7) and (8.8) we obtain

$$
\sum_{\mu \in \mathrm{WComp}_{n, m}} F_{\mu}=\sum_{k=0}^{n-1}\left(\begin{array}{c}
n-1 \\
k
\end{array}\right) t^{k} \sum_{w \in[m]^{n-k}} t^{\operatorname{des}(w)} .
$$

Now set $m=n+1$ and plug this equation into (8.6) to obtain the desired result (8.2).

Proof of (8.3). It follows from (8.1) and (3.2) that

$$
\beta\left(\mathrm{NC}_{n+1}^{-} * T_{t, n-1}\right)=\sum_{\mu \in \mathrm{PC}_{n}} G_{\mu}
$$

where

$$
G_{\mu}:=\sum_{\substack{w \in \mathfrak{S}_{M(\mu)} \cap \mathrm{NDD}_{n} \\ w_{n-1} \leq w_{n} \\ w_{1} \leq w_{2}}} t^{\operatorname{des}(w)+1}(t+1)^{n-2 \operatorname{des}(w)-2} .
$$

Using a similar argument to that which was used to derive (8.6) (with (4.3) now playing the role of (4.4)) we obtain

$$
\beta\left(\mathrm{NC}_{n+1}^{-} * T_{t, n-1}\right)=\frac{1}{n+1} \sum_{\mu \in \mathrm{WComp}_{n, n+1}} G_{\mu}
$$

For $n \geq 1$, let

$$
F_{n}:=\sum_{\substack{w \in \mathrm{NDD}_{n} \\ w_{n-1} \leq w_{n}}} t^{\operatorname{des}(w)}(1+t)^{n-1-2 \operatorname{des}(w)} \mathbf{x}_{w}
$$


and

$$
G_{n}:=\sum_{\substack{w \in \mathrm{NDD}_{n} \\ w_{1} \leq w_{2} \\ w_{n-1} \leq w_{n}}} t^{\operatorname{des}(w)}(1+t)^{n-2-2 \operatorname{des}(w)} \mathbf{x}_{w} .
$$

Also let $F_{0}=G_{0}=1$. By (4.4) and (4.3) we have

$$
\sum_{n \geq 0} F_{n} z^{n}=\sum_{n \geq 0} G_{n} z^{n} \sum_{n \geq 0} h_{n} z^{n} .
$$

Hence

$$
\sum_{n \geq 0} G_{n} z^{n}=\sum_{n \geq 0} F_{n} z^{n} \sum_{n \geq 0}(-1)^{i} e_{n} z^{n} .
$$

Equating coefficients of $z^{n}$ yields

$$
G_{n}=\sum_{r=0}^{n}(-1)^{r} e_{r} F_{n-r}
$$

By applying to (8.11), the specialization that sets

$$
x_{i}= \begin{cases}1 & \text { if } i \in[n+1] \\ 0 & \text { otherwise }\end{cases}
$$

we obtain

$$
\sum_{\mu \in \mathrm{WComp}_{n, n+1}} G_{\mu}=(-1)^{n}(n+1)+\sum_{r=0}^{n-1}(-1)^{r}\left(\begin{array}{c}
n+1 \\
r
\end{array}\right) \sum_{\mu \in \mathrm{WComp}_{n-r, n+1}} F_{\mu} .
$$

By plugging (8.9) into this equation we obtain

$$
\begin{aligned}
\sum_{\mu \in \mathrm{WComp}_{n, n+1}} G_{\mu}= & (-1)^{n}(n+1)+\sum_{r=0}^{n-1}(-1)^{r}\left(\begin{array}{c}
n+1 \\
r
\end{array}\right) \sum_{k=0}^{n-r-1}\left(\begin{array}{c}
n-r-1 \\
k
\end{array}\right) t^{k} \\
& \times \sum_{w \in[n+1]^{n-r-k}} t^{\operatorname{des}(w)} .
\end{aligned}
$$

The desired result (8.3) follows from this and (8.10). 


\section{References}

[1] R. Askey and M. Ismail (1976). Permutation problems and special functions. Canad. J. Math. 28 853-894. MR0406808

[2] A. Björner (1980). Shellable and Cohen-Macaulay partially ordered sets. Trans. AMS 260 159-183. MR0570784

[3] A. Björner, A. Garsia, and R. P. Stanley (1982). An introduction to the theory of Cohen-Macaulay posets. In: Ordered Sets, I. Rival, ed., Reidel, Dordrecht/Boston/London, pp. 583-615. MR0661307

[4] A. Björner and M. L. Wachs (1982). Bruhat order of Coxeter groups and shellability. Advances in Math. 43 87-100. MR0644668

[5] A. Björner and M. L. Wachs (1983). On lexicographically shellable posets. Trans. AMS 277 323-341. MR0690055

[6] A. Björner and M. L. Wachs (1996). Nonpure shellable complexes and posets I. Trans. AMS 348 1299-1327. MR1333388

[7] A. Björner and V. Welker (2005). Segre and Rees products of posets, with ring-theoretic applications. J. Pure Appl. Algebra 198 43-55. MR2132872

[8] L. Butler (1994). Subgroup lattices and symmetric functions. Memoirs of the Amer. Math. Soc. 112. MR1223236

[9] L. Carlitz, R. Scoville, and T. Vaughan (1976). Enumeration of pairs of sequences by rises, falls and levels. Manuscripta Math. 19 211-243. MR0432472

[10] D. Foata and M.-P. Schützenberger (1970). Théorie géométrique des polynomes eulériens, Lecture Notes in Mathematics 138, SpringerVerlag, Berlin-New York. MR0272642

[11] I. M. Gessel and M. L. Wachs. $\gamma$-positivity of a symmetric function analog of the Eulerian polynomials, in preparation.

[12] J. Jonsson. The Rees product of a Boolean algebra and a chain, preprint.

[13] D. Kim and J. Zeng (2001). A new decomposition of derangements. J. Combin. Theory Ser. A 96 192-198. MR1855792

[14] G. Kreweras (1972). Sur les partitions non croisées d'un cycle. Discrete Math. 1 333-350. MR0309747 
[15] P. A. MacMahon (1960). Combinatory Analysis, 2 volumes, Cambridge University Press, London, 1915-1916. Reprinted by Chelsea, New York. MR0141605

[16] P. A. MacMahon (1913). The indices of permutations and the derivation therefrom of functions of a single variable associated with the permutations of any assemblage of objects. Amer. J. Math. 35(3) 281-322. MR1506186

[17] P. Muldoon Brown and M. A. Readdy (2011). The Rees product of posets. Journal of Combinatorics 2 165-191. MR2913192

[18] D. Rawlings (1981). The r-major index. J. Combin. Theory, Ser. A 31 175-183. MR0629592

[19] J. Shareshian and M. L. Wachs (2007). q-Eulerian polynomials: Excedance number and major index. Electron. Res. Announc. Amer. Math. Soc. 13 33-45. MR2300004

[20] J. Shareshian and M. L. Wachs (2010). Eulerian quasisymmetric functions. Advances in Math. 225 2921-2966. MR2728998

[21] J. Shareshian and M. L. Wachs (2009). Poset homology of Rees products and q-Eulerian polynomials. Electronic J. Combinatorics 16 R20. MR2576383

[22] J. Shareshian and M. L. Wachs (2012). Chromatic quasisymmetric functions and Hessenberg varieties. In: Configuration Spaces, Proc., A. Björner, F. Cohen, C. De Concini, C. Procesi and M. Salvetti, eds., Edizioni della Normale, Pisa, pp. 433-460. (arXiv:1106.4287)

[23] J. Shareshian and M. L. Wachs. Chromatic quasisymmetric functions, in preparation.

[24] R. P. Stanley (1983). Combinatorics and Commutative Algebra, 2nd ed., Progress in Mathematics 41, Birkhäuser, Boston, 1996. MR1453579

[25] R. P. Stanley (1995). A symmetric function generalization of the chromatic polynomial of a graph. Advances in Math. 111 166-194. MR1317387

[26] R. P. Stanley (1996). Parking functions and noncrossing partitions. Wilf Festschrift, Electronic Journal of Combinatorics 3 \# R20. MR1392491

[27] R. P. Stanley (1997). Enumerative Combinatorics, Vol. 1, 2nd ed., Cambridge Studies in Advanced Mathematics 49, Cambridge University Press, Cambridge. MR1442260 
[28] M. L. Wachs (2007). Poset topology: Tools and applications. In: Geometric Combinatorics, IAS/PCMI Lecture Notes Series 13, E. Miller, V. Reiner, B. Sturmfels, eds., pp. 497-615. MR2383132

Svante Linusson

DEPARTMENT OF MathEMATICS

KTH-Royal Institute of TEChNOLOGY

SE-100 44, STOCKHOLM

SWEDEN

E-mail address: linusson@math.kth.se

JoHn SHARESHIAN

DEPARTMENT OF Mathematics

WASHINGTON UNIVERSITY

St. Louis, MO 63130

USA

E-mail address: shareshi@math.wustl.edu

Michelle L. WAChS

DEPARTMENT OF Mathematics

University OF Miami

Coral Gables, FL 33124

USA

E-mail address: wachs@math.miami.edu

RECEIVED JANuARY 31, 2012 